\title{
An omni-RGB+D camera rig calibration and fusion using unified camera model for 3D reconstruction (Withdrawal Notice)
}

Ahmad Zawawi Jamaluddin, Osama Mazhar, Cansen Jiang, Ralph Seulin, Olivier Morel, et al.

Ahmad Zawawi Jamaluddin, Osama Mazhar, Cansen Jiang, Ralph Seulin, Olivier Morel, David Fofi, "An omni-RGB+D camera rig calibration and fusion using unified camera model for 3D reconstruction (Withdrawal Notice)," Proc. SPIE 10338, Thirteenth International Conference on Quality Control by Artificial Vision 2017, 1033809 (14 May 2017); doi: 10.1117/12.2266945

SPIE Event: The International Conference on Quality Control by Artificial Vision 2017, 2017, Tokyo, Japan 


\section{An omni-RGB+D camera rig calibration and fusion using unified camera model for $3 D$ reconstruction}

Ahmad Zawawi Jamaluddin, Osama Mazhar, Cansen Jiang, Ralph Seulin, Olivier Morel and David Fofi Lab. Electronique, Informatiique et Image, Univ. Bourgogne France-Comté, CNRS (France)

Proc. SPIE 10338, 1033809 (2017)

Online Publication Date: 14 May 2017

Withdrawn from Publication: 17 May 2017

Conference Date: 14-16 May 2017

Conference Location: Tokyo, Japan

Conference Title: The International Conference on Quality Control by Arificial Vision 2017

Conference Chairs: Hajime Nagahara, Kazunori Umeda, and Atsushi Yamashita

This paper has been withdrawn by the publisher because it was not presented at the conference.

Thirteenth International Conference on Quality Control by Artificial Vision 2017, edited by Hajime Nagahara,

Kazunori Umeda, Atsushi Yamashita, Proc. of SPIE Vol. 10338, 1033809 • (c) 2017 SPIE

CCC code: $0277-786 X / 17 / \$ 18 \cdot$ doi: $10.1117 / 12.2266945$ 